\title{
Intergenerational Groups: Rediscovering our Legacy
}

\author{
Scott P. Anstadt \\ Deb Byster
}

\begin{abstract}
Intergenerational groups are a community-based group concept designed to engage and mobilize often untapped resources of older adults in effective interaction with younger populations. These groups support an atmosphere of synergistic interaction. Members of each generation share reflections on interpersonal strengths and capacities and rediscover emotional and spiritual anchors and bonding. Illustrated here is Community Connections (CC), developed using the phase driven participatory culturespecific intervention model (PCSIM; Nastasi, Moore \& Varjas, 2004) that included self selected local older adults, caregivers, and multicultural exchange students. The program was structured to offer mutual opportunities for activities built around exchanging cultural and life experiences. The goals were: 1) to reduce social isolation due to age, culture, or disability 2) for international students to practice English and learn about local cultural traditions, and 3) to build intergenerational 'extended family' relationships.
\end{abstract}

Keywords: Intergenerational; community outreach; group; older adult; international students; multicultural

\section{INTRODUCTION}

Intergenerational groups connote the process of bridging beyond and moving across what is often perceived as barriers in relationships between generations. Several studies support the benefits of systematically reducing self isolation for both older adults and other vulnerable populations and a strong and consistent social support system which is offered to the consumer (outreach) reduces the risk of inpatient medical and mental health hospital utilizations, chronic physical illnesses such as cardiovascular diseases, and the need for long term out of home placements (Caplan, 2004; Chan, Kasper, Black \& Rabins, 2003; Ghose, Williams \& Swindle, 2005; Keyes, 2004; 2005; Reynolds et al., 1997). The benefits of psychosocial contact in helping to prevent depression (e.g. Kasen, Cohen, Chen \& Castille, 2003; Russell \& Cutrona, 1991) and anxiety (e.g. Fry, 2003) as well as improving overall quality of life (e.g., Cohen \& Wills, 1985; House, Landis \& Umberson, 1988; Stanley, Beck \& Zebb, 1998) have been well researched and documented.

Programs supplying these intergenerational social support services serve as ice breakers' which encourage the participants to venture out of their home more often to seek additional services such as pharmacological and/or psychosocial interventions, or to enjoy the companionship of others (Health Resources and Services Administration, 1993; Korte \& Gupta, 1988; Mistry, Rosansky, McGuire, McDermott \& Jarvik, 2001). Common

Scott P. Anstadt, Ph.D, DCSW, LCSW, IABMCP, is an instructor in the Division of Social Work, College of Professional Studies, Florida Gulf Coast University, Fort Myers, Fl. Deb Byster is a Master's candidate at Walden University.

Copyright (C) 2009 Advances in Social Work Vol. 10 No. 1 (Spring 2009), 39-50 
Common to all of these studies is that positive informal interactions and trustworthy companionship result in higher self-efficacy, feelings of well being, and self-esteem (Bartels et al., 2002; Bruce, Van Citters \& Bartels, 2005; Cole \& Dendukuri, 2003; Cuijpers, 1998).

More specifically, this community-based group concept is designed to engage and mobilize the considerable and often untapped resources of the older adult population in effective and sharing interaction with targeted younger populations. The younger populations may consist of cohorts of various age ranges, i.e. toddlers, children, adolescents, or younger adults. The focus of activities and interactions is strength-based, leading to both recognition of what each generation can offer to a group activity and what each may learn in the process (University of Pittsburgh, n.d.). Transformation away from a negative thought framework is commandeered through the development of mutual appreciation of commonality and generativity at all ages. Members become both mentors and learners alike in a holographic context whereby members solidify self concept through reciprocal positive regard. (Mistry et al., 2001).

The literature shows these groups to be primarily open-ended and based in a community setting such as daycare settings, schools, senior centers, houses of worship, and recreational centers. The structure and theoretical orientation of the group sets the stage for an atmosphere of synergistic interaction (Yang \& Jackson, 1998); thus microcosmic development of a sense of community can be generalized to applications in everyday life. Each generation looks at interpersonal strengths and capacities in a contrapuntal and harmonious discovery of ways to relate across a generation gap from which they may have previously felt cast adrift and segregated (Ghose et al., 2005; Keyes, 2004; 2005; Reynolds et al., 1997). Self-efficacy around having purpose and a place in humanity expands member perceptual, cognitive, spiritual, and social boundaries and offsets perceptions of aloneness, obsolescence, and self isolation. Examples of activities include: a wellness education focus, caregiver supports, sharing vocational expertise and skills, life story reminiscence, parenting skill building (such as foster grandparents), tutoring school work, crafts, and volunteerism (Seidel, Smith, Hafner \& Holme, 1992; Vitaliano, 1995).

Using the medium of narrative self expression or 'storytelling' in its many natural forms, members come to appreciate and understand generational idiosyncrasies of expression around group tasks and reminiscent sharing, thus encouraging members to stretch themselves into a deeper appreciation of generational cultural diversity. Mutual benefits are found in reducing age and culturally based stereotypes, building mutual respect, and increasing understanding of each other's generations through 'life story' reminiscence and mentoring (Kwan, Love, Ryff \& Essex 2003; LaPorte, 1999).

A spiritual appreciation for all persons and accompanying lightness of mood in the group is often the product of this process (Aranda, 1990). Mutual assistance in both expressive and task oriented skill building can kindle faith, appreciation, and conviction in a common foundation for all humanity. Cultural traditions and legacy can be passed on through positive reflective interaction and trustworthy companionship. Such a cyclical mirror effect has lead to self evaluative ratings showing increased self-efficacy, sense of 
well being, and self-esteem (Kwan et al., 2003; McGowan, 1994; Morris, 1984; Raynes, 2003). In this regard, these community-based groups have primary and secondary prevention potential (Health Resources and Services Administration, 1993; Korte \& Gupta, 1988).

\section{ILLUSTRATIVE PROGRAM - COMMUNITY CONNECTIONS}

Community Connections (CC) was launched in spring 2007 as a pilot program that included self-selected international exchange and multicultural students, self-selected older adults and caretakers, Center for Life Experiences (a faith based community outreach program) staff, and community volunteers. CC was held every other Friday beginning in the later afternoon and extending into the early evening. The program was structured to offer mutual opportunities for activities built around exchanging cultural and life experiences through the fine arts and small narrative story telling groups. It provided a "community" family style meal that included nutritionally healthy foods of both local and international cuisines. The goals of this community-based, intergenerational group program were : 1) to reduce the social isolation of older adults, caregivers, and international and multicultural students and offer them the opportunity to get out of a limited social environment due to age, culture, or confinement, caring for a family member or declining health, 2) to expand the opportunities for international and multicultural students to practice their English and learn more about local cultural and American traditions through interaction with community members in a social nonacademic setting, and 3) to build relationships between older adults needing to feel valued through sharing their life experiences and international students who are seeking the security of "extended family" relationships found in many of their cultures.

\section{Background, Rationale and Significance of Program Framework}

$\mathrm{CC}$ was a community-based program, providing a gathering place for individuals to convene every other week. The First Presbyterian Church provided the space at no charge and placed no requirements on the $\mathrm{CC}$ groups. A room large enough to hold up to 100 participants had a kitchen for meal sharing. Transportation through the generous support of the university was provided to the site for those unable to provide their own. The theme, structure of presentations and activities as well as the meal presentations were developed in monthly CC Task Force meetings. These were attended by representatives from a variety of community and university-based groups facilitated by a part time Director of CC.

The meeting lasted approximately three hours and included a culturally-based presentation and activity with participant involvement. This activity, as with so many culturally-based traditions, was an example of a universal theme with many counterparts throughout the world. Dance and music was one such example. At various meetings, ethnic student and local resident groups would share their music and teach participants how to enjoy simple dance moves. CC participants may experience polka in one meeting, tai chi in another, and rap in yet a third. These presentations of universal themes 
illustrated through culturally-based activities provided a focus for conversation during a buffet style dinner enjoyed at small tables. Here, for example, favorite music and dances participants do could be shared in dialogue. Staff facilitators at these tables encouraged a narrative exchange of ideas. Folks were encouraged to 'tell their story' about the universal theme. This rich sharing of past and present experiences encouraged deeper interaction where folks could relate to common themes, and emotions. This, according to many of the participants, seemed to help build continuing relationships. For example, many of the international students and older adults 'adopted' each other by keeping in touch by phone and regular visits.

\section{Population Groups Served}

Older adults 65 years of age and older represent the fastest growing segment of our population, about one half of which live below the Federal poverty line, one out of three lives alone, and close to one half are disabled (National Family Caregivers Association (2006). Most older adults have traditionally relied upon informal support networks such as families which in recent years have been dwindling due to out-migration (U. S. Census Bureau, 2000; Connelly, 2002; Glasser et al., 2003; Markello, 2002; McDonald, 2001).

Caregivers of the older adults are also in need of social support, education, and social service coordination assistance. A recent research study conducted jointly by the National Association of Social Workers and the New York Academy of Medicine (2008) described caregivers as the 'sandwich generation' referring to the stress related to taking care of and providing for both children and elderly parents (Family Caregiver Alliance, 2006). International exchange students, through the local university International Partners program, were a motivating force in the CC program. They came a long way to learn both the English language as used in fluid and colloquial conversation and the rich subtleties of a local U.S. culture. Participation in local and down home activities within a helping context such as CC represented a very essential part of international students' experience (Barker \& Smith, 1996; Harvey, 2007).

Targeted older adults, caregivers, and the multicultural students were somewhat isolated from the rest of the community; the older adults and caregivers by the physical conditions and limitations, and the students by their language skills and marginalized status in the predominantly European American community.

\section{Program Theoretical Model}

The conception of the program started with a strong conviction in the strengths-based foundation of community-based outreach groups (Carruthers, Hood \& Parr, 2005). Such a foundational approach allows application of a social exchange enhancement process designed to give isolated older adults, caregivers, and multicultural/international students in a rural community an opportunity to transcend their traditional social networks, and engage in a new and thought provoking interactive environment.

The participatory culture-specific intervention model (PCSIM), a community planning approach, was used in the inception and developmental phases of the CC 
intergenerational group program. PCSIM is helpful in facilitating achievement of both individual and sociocultural (transcultural) goals related to the mental health of the participant generational groups. PCSIM methodology provides an intervention model for translating mental health research into practice while incorporating the views of culturally diverse participants, providing a guideline for developing empirically based and culturally specific services (Nastasi, Moore \& Varjas, 2004). The following outlines with examples how this model was used in phase development of CC.

Phase 1: Existing theory, research, and practice. The initial germ of an idea for CC came from students and staff at the university. This raised the question of what had been done in other communities where such a need existed. A literature review resulted. This lead to the selection of the PCSIM approach as described here. Nested within this approach is our integration of the Values in Action (Peterson \& Seligman, 2003) framework of service to the community with reciprocal beneficial qualities. This is essentially a strength-based approach as applied to community action development using an adult education model of resident participation in building community capacity through organizing and networking local groups.

Phase 2: Learning the culture. Initial efforts concentrated on discovering what types of programs were currently being offered in the community which proactively addressed the mental health of older adults, caregivers and multicultural groups and whose aim was to strengthen diversity and multicultural acceptance.

In addressing the multicultural aspects of the target populations, individuals with cultural expertise were approached to contribute to the early concept development of CC. The CC task force concluded there was a gap in services that could be provided simultaneously and in a synergistic manner to persons of various generations and cultural backgrounds. The university had its student International Partnership organizations and the local community had various community-based groups including various houses of worship and the Area Agency on Aging, but these groups had no activities in common and so remained segregated from each other.

Phase 3: Forming partnerships. The CC Program Director assumed the responsibility of recruiting the $\mathrm{CC}$ task force with intention to fully involve community grass roots efforts. The participants of the task force were recruited through informal networking of key stakeholder groups in the community. These included community agencies, faithbased groups, community action groups, adult education, the university student coalition of groups, and the university administration. This allowed for publicity to be distributed throughout the community and a resulting growing buy-in of interest. For each of these groups, the extended commitment and involvement was negotiated. For example, a letter of support for the program's development was secured from the university. This allowed $\mathrm{CC}$ to advertise on campus and to secure transportation through the university administration.

Phase 4: Goal or problem identification. At the first task force meeting, findings of empirical research gathered in Phases 1 and 2 were presented. Agreement that the program was of benefit was solicited and received. Discussion ensued regarding how each 
each team member's expertise and interests could enhance the development of this program, and what efforts they could bring to the program to help ensure its success.

An important part of this process is the continual refinement of consensus on the goals and general methods. It is in this identification phase that problem-solving skills were refined and normed. In this manner divergent ideas could be integrated. This process turned out to be as critical to the development of CC as the content of the CC meetings; it was the glue that held the whole project together.

Phase 5: Formative research. Additional constituent organization representatives were identified and encouraged to join the task force. Some focus groups were formed in order to solicit feedback on potential $\mathrm{CC}$ activities to meet the primary goal of cultural sharing and enrichment within the community. For example, $\mathrm{CC}$ had a presentation on the universal theme of holiday celebrations featuring the Chinese New Year. For this to be truly meaningful, a focus group was formed headed by the Chinese Student Association. The purpose was to explore amongst themselves what that holiday season really meant to them. They went on to speak to various other student and community groups to get a sense of which activities could have associated meaning for Americans.

Phase 6: Culture-specific theory or model. The culture-specific model was developed as a specific agenda item in task force planning meetings for each CC. Based on targetspecific formative research such as used above, the considerations of why one method was chosen over another were refined. Additional experts and stakeholders from the community were also identified to add further refinement where possible. For example, the preparation of the meals was done in an ethnic manner. However expert opinion had to be gleaned as to how various tastes and appetites might appreciate certain dishes.

Phase 7: Program design (participatory generation). Specific component activities for the groups were identified and began to take tangible form. This was a creative and at times frustrating phase with competing ideas that needed to be discussed and coalesced. It was necessary to form sub-committees and establish priorities.

CC did not have the structure of programming described above at its inception. The format developed over time and with many intense and committed discussions of the task force. Sticking with this process allowed the CC program structure to solidify. Perhaps one of the most conscious group normative developmental aspects in all phases was the encouragement of all task force participants to feel welcome and appreciated for their contributions.

Phase 8: Program implementation (natural adaptation). Any proposed alterations in the program needed to be evaluated to ensure that enough was known about the shortcoming and determine whether a component could be enhanced (such as through training). Since the $\mathrm{CC}$ task force was comprised of stakeholders, team building required continual affirmative buy-in to the CC program as it was evolving, including resolving subtle issues. One example involved the length of time for the CC meetings. Since both young adults and older folks were involved, a balance of time and energy had to be found. The task force elicited comment from the participants and came up with the three-hour program. 
Phase 9: Program evaluation (essential changes and elements). In the development of the evaluation plan, we were careful to identify the goals stated at the project's inception, and to associate various stakeholders with each of those goals. By doing this, we were able to identify the appropriate parties to provide their estimation of the success of each goal as well as the overall program.

\section{Pilot Study Data Collection}

Over the course of the first year and one-half of the CC program, a pilot study was conducted through the use of a simple four question satisfaction survey handed to the participants at the end of each meeting and completed before they left. The questions asked if participants felt the atmosphere welcomed social and cultural exchange provided an opportunity to meet new friends, share culturally based traditions and activities, and provided an opportunity for the international students to practice conversational English. Collection included 400 student and 364 community participant surveys representing $69.6 \%$ of all participants who attended. Students represented 21 nations with the highest number from China. The local older adult community ranged in age from 57 to 103 . The data were used to give feedback to the Task Group as it was formulating and developing the $\mathrm{CC}$ program. The descriptive research question explored if $\mathrm{CC}$ provided an opportunity of positive social exchange, defined as creating a welcoming atmosphere, opportunity to meet potential friends, and share culturally based activities amongst younger and older adults from a variety of cultural backgrounds. Results are shown in Table 1.

We plan to conduct interviews and focus groups with selected participants (and nonparticipants) to better understand their qualitative evaluation of the program or reasons for non-participation. It is hoped that our surveys in combination with these interviews and focus groups will provide data on which decisions may be made to monitor and improve the program in the future. 
Table 1: $\quad$ Percent of Participants Endorsing Social Exchange Opportunities at Community Connections as a Function of Participant Type

\begin{tabular}{lcccc} 
& & Social Exchange Items & \\
Participant type & $\begin{array}{c}\text { Welcoming } \\
\text { atmosphere }\end{array}$ & $\begin{array}{c}\text { Opportunity } \\
\text { to meet } \\
\text { friends }\end{array}$ & $\begin{array}{c}\text { Cultural } \\
\text { exchange }\end{array}$ & $\begin{array}{c}\text { Practice } \\
\text { conversational } \\
\text { English }\end{array}$ \\
\hline International students & $100 \%$ & $96 \%$ & $98 \%$ & $97 \%$ \\
Older adults and caregivers & $99 \%$ & $98 \%$ & $98 \%$ & N/A \\
\hline
\end{tabular}

\section{Intervention: Program Continuation or Extension}

Phase 10: Capacity building (sustainability and institutionalization). The decisionmaking involvement of our stakeholders at every step has contributed to their sense of ownership, as well as their continued interest and involvement, and serves to empower them and sustain the program. As we are better known in the community, we have grown. Donations and materials for the meetings are more available, allowing the programming to be more sophisticated. However, as new partnerships of support are developed, it is necessary to be creative concerning how the program can benefit them directly and to welcome the increased range of interest in program agenda. For example, a CC program on Kwanza now involves not just the Black Student Union but also several local religious and cultural organizations. Its presentation involves a wide variety of traditions.

Phase 11: Translation (dissemination and deployment). We do have plans in the near future to implement a next stage of the program which will involve group support for caregivers, as well as in-home visits as a vehicle for service learning experience for university students in the behavioral sciences (Social Work, Counseling and Psychology). These may include the gleaning of local oral histories.

\section{CONCLUSION}

Community Connections, an intergenerational and multicultural strength-based community outreach group, used the application of PCSIM throughout its development, implementation and ongoing evaluation. At each developmental phase of the PCSIM model, a facilitated team-building approach sought out and honored the considerable input of various task force stakeholder groups to craft each $\mathrm{CC}$ community event. The task force has been a model of the intergenerational group process enlightenment. Dedication to the viability of the program's goals has transcended all differences and continues to shine a light of faith on opportunities for personal growth and interpersonal community-based cohesiveness. 


\section{References}

Aranda, M. P. (1990). Culture-friendly services for Latino elders. Journal of the American Society on Aging, 14, 55-57.

Barker, T. S., \& Smith, H. W. (1996). A perspective of a new taxonomy for international education. International Education, 26, 40-55.

Bartels, S. J., Dums, A. R., Oxman, T. E., Schneider, L. S., Alexopoulos, G. S., \& Jeste, D. (2002). Evidence-based practices in geriatric mental health care. Psychiatric Services, 53, 1419-1431.

Bruce, M. L., Van Citters, A. D., \& Bartels, S. J. (2005). Evidence-based mental health services for home and community. Psychiatric Clinics of North America, 28, 10391060 .

Caplan, G. (2004). A randomized, controlled trial of comprehensive geriatric assessment and multidisciplinary intervention after discharge of elderly from the emergency department-The Deed II study. Journal of the American Geriatrics Society, 52, $1417-1423$.

Carruthers, C., Hood, C. D., \& Parr, M. (2005). Research update: The power of positive psychology. Parks \& Recreation, 40, 30-37.

Chan, D. C., Kasper, J. D., Black, G. S., \& Rabins, P. V. (2003). Presence of behavioral and psychological symptoms predicts nursing home placement in communitydwelling elders with cognitive impairment in univariate but not multivariate analysis. Journals of Gerontology: Series A: Biological Sciences and Medical Sciences, 58A, 548-554.

Cohen, S., \& Wills, T. A. (1985). Stress, social support, and the buffering hypothesis. Psychological Bulletin, 98, 310-357.

Cole, M. G., \& Dendukuri, N. (2003). Risk factors for depression among elderly community subjects: A systematic review and meta-analysis. American Journal of Psychiatry, 160, 1147-1156.

Connelly, L. (2002). Rural Kansas. Kansas eldercount. New York: Milbank Memorial Fund. [Electronic Version] Retrieved on January 7, 2007 from http://www2.kumc.edu/coa/Education/AMED900/Population.pdf

Cuijpers, P. (1998). Psychological outreach programmes for the depressed elderly: A meta-analysis of effects and dropout. International Journal of Geriatric Psychiatry, 13, 41-48.

Family Caregiver Alliance. (2006). Caregiving Statisitics. Retrieved February 1, 2007 from National Family Caregivers Association: http://www.nfcacares.org/who are family_caregivers/care_giving_statstics.cfm

Fry, P. S. (2003). Perceived self-efficacy domains as predictors of fear of the unknown and fear of dying among older adults. Psychology and Aging, 18, 474-486. 
Ghose, S. S., Williams, L. S., \& Swindle, R. W. (2005). Depression and other mental health diagnoses after stroke increase inpatient and outpatient medical utilization three years post stroke. Medical Care, 43, 1259-1264.

Glasser, M., Holt, N., Hall, K. Mueller, B. Norem, J., Pickering, et al. (2003). Meeting the needs of rural populations through interdisciplinary partnerships. Family \& Community Health, 26, 230-45.

Harvey, D. A. (2007). A preference for equality: Seeking the benefits of diversity outside the educational context. BYU Journal of Public Law, 21, 55-82

Health Resources and Services Administration (1993). Rural health: The story of outreach. A program of cooperation in health care. Rockville, MD: [Author].

House, J. S., Landis, K. R., \& Umberson, D. (1988). Social relationships and health. Science, 241, 540-545.

Kasen, S., Cohen, P., Chen, H., \& Castille, D. (2003). Depression in adult women: Age changes and cohort effects. American Journal of Public Health, 93, 2061-2066.

Keyes, C. L. M. (2004). Nexus of cardiovascular disease and depression revisited: The complete mental health perspective and the moderating role of age and gender. Aging and Mental Health, 8, 266-274.

Keyes, C. L. M. (2005). Chronic physical conditions and aging: Is mental health a potential protective factor? Ageing International, 30, 88-104.

Korte, C., \& Gupta, V. (1988, November). The relational needs of single elderly: A test of competing hypotheses. Paper presented at the Annual Meeting of the Gerontological Society, San Francisco, CA.

Kwan, C. M. L., Love, G. D., Ryff, C. D., \& Essex, M. J. (2003). Role of self-enhancing evaluations in a successful life transition. Psychology and Aging, 18, 3-12.

LaPorte, A. M. (1999, March). Building community through intergenerational art education. Paper presented at the annual meeting of the National Art Education Association, Washington, DC.

McDonald, A. (2001). Staying home alone - Working in the community with older people who have dementia. Brighton: Pavilion Publishing Ltd.

McGowan, T. G. (1994). Mentoring-reminiscence: A conceptual and empirical analysis. International Journal of Aging \& Human Development, 39, 321-36.

Markello, S. (2002). Health. Kansas eldercount. New York: Milbank Memorial Fund. [Electronic Version] Retrieved on January 7, 2007 from http://www2.kumc.edu/coa/Education/AMED900/Health.pdf

Mistry, R., Rosansky, J., McGuire, J., McDermott, C., \& Jarvik, L. (2001). Social isolation predicts re-hospitalization in a group of older American veterans enrolled in the UPBEAT program. International Journal of Geriatric Psychiatry, 16, 950-959.

Morris, J. H., Jr. (1984, July). Project teen-ager - A skills exchange program: High school 
school students volunteering with the elderly in a rural community. Paper presented at the meeting of the National/International Institute on Social Work in Rural Areas, Orono, ME.

NASW; The New York Academy of Medicine. (2008). Surveying the Sandwich Generation Women 2006. Retrieved March 31, 2008 from National Association of Social Workers: http://www.helpstartshere.org/Default.aspx?PageID=1342

Nastasi, B. K., Moore, R. B., \& Varjas, K. M. (2004). School-based mental health services: Creating comprehensive and culturally specific programs. Washington DC: American Psychological Association.

Peterson, C., \& Seligman, M. E. P. (2003). The Values in Action (VIA) classification of strengths. Washington, DC: American Psychological Association.

Raynes, N. V. (2003). Intergenerational programmes: What are they? Why are they there? Where are they going? Intercom, 10, 5-7.

Reynolds, III, C. F., Frank, E., Houck, P.R., Mazumdar, S., Dew, M.A., Cornes, C., Buysse, D. J., et al. (1997). Which elderly patients with remitted depression remain well with continued interpersonal psychotherapy after discontinuation of antidepressant medication? American Journal of Psychiatry, 154, 958-962.

Russell, D. W., \& Cutrona, C. E. (1991). Social support, stress, and depressive symptoms among the elderly: Test of a process model. Psychology \& Aging, 6, 190-201.

Seidel, G., Smith, C., Hafner, R. J., \& Holme, G. (1992). A psychogeriatric community outreach service: Description and evaluation. International Journal of Geriatric Psychiatry, 7, 347-350.

Stanley, M. A., Beck, J. G., \& Zebb, B. J. (1998). Psychometric properties of the MSPSS in older adults. Aging and Mental Health, 2, 186-193.

U.S. Census Bureau (2000). Population estimates: Geographies ranked by estimate. Retrieved on April 13, 2007 from http://factfinder.census.gov/servlet/GCTTable? bm=y\&-context=gct\&ds name $=$ PEP 2008 EST\&-mt name $=$ PEP 2008 EST GCTT1R ST2S\&CONTEXT $=$ gct $\&$-tree $\mathrm{id}=808 \&$-redoLog $=$ true $\&$-geo $\mathrm{id}=04000 \mathrm{US} 20 \&$-format $=$ ST$\underline{2 \mid S T-2 S \&-\text { lang }=\text { en }}$

University of Pittsburg: Generations Together-An Intergenerational Studies Program. (n.d.). Retrieved on November 26, 2008 from http://www.gt.pitt.edu

Vitaliano, P. P. (2005). Making the case for caregiver research in geriatric psychiatry. American Journal of Geriatric Psychiatry, 13, 834-843.

Yang, J. A., \& Jackson, C. L. (1998). Overcoming obstacles in providing mental health treatment to older adults: Getting in the door. Psychotherapy, 35, 498-505. 


\section{Author's note:}

Address correspondence to: Scott P. Anstadt, Ph.D., Division of Social Work, College of Professional Studies, Florida Gulf Coast University, Fort Myers, FL 33907.

Email: revscottaz@yahoo.com or sanstadt@,fgcu.edu 